\title{
Tumor cell migration is inhibited by a novel therapeutic strategy antagonizing the alpha-7 receptor
}

\author{
Chris Pepper ${ }^{1}$, Henry Tu², Paul Morrill², Sara Garcia-Rates², Chris Fegan ${ }^{1}$, Susan \\ Greenfield ${ }^{2}$ \\ ${ }^{1}$ Division of Cancer and Genetics, Cardiff University School of Medicine, Cardiff, CF14 4XN, UK \\ ${ }^{2}$ Neuro-Bio Ltd, Culham Science Centre, Abingdon, OX14 3DB, UK \\ Correspondence to: Susan Greenfield, email: Susan.greenfield@neuro-bio.com \\ Keywords: alpha-7 receptor, acetylcholinesterase peptide, cyclized variant, metastases, cell migration
}

Received: July 06, $2016 \quad$ Accepted: December 26, 2016

Published: January 06, 2017

\section{ABSTRACT}

A 14mer peptide (T14) derived from the C-terminus of acetylcholinesterase (AChE) selectively activates metastatic breast cancer cells via the alpha-7 nicotinic receptor ( $\mathbf{A} 7 \mathrm{nAChR}$ ). This naturally occurring peptide is also present in brain, is elevated in Alzheimer's disease, and is antagonised by a cyclized variant (NBP-14). Here we investigated the effects of NBP-14 in six different cancer cell lines, primary leukemia B-cells and normal B-cells. All cells tested expressed a7 nAChR, intracellular and extracellular T14. However, NBP-14 showed low toxicity and weak antiproliferative effects in the majority of the cell lines and was even less toxic in normal B-cells when compared to primary chronic lymphocytic leukemia cells $(P<0.001)$. Given the potential role of $\mathrm{T} 14$ peptide in metastasis, we next investigated the effects of NBP-14 on tumor cell migration, where it caused a dose-dependent reduction. The extent of NBP-14 inhibition positively correlated with the migration of the cells $\left(r^{2}=0.45 ; P=0.06\right)$. Furthermore, NBP-14 preferentially inhibited the migration of primary leukemia cells when compared with normal B-cells $(P=\mathbf{0 . 0 0 0 2})$; when the normal B-cell data was excluded, this correlation was strengthened $\left(r^{2}=0.80\right.$; $P=0.006$ ). Importantly, the constitutive a7 nAChR expression positively correlated with intracellular T14 levels $\left(r^{2}=0.91 ; P=0.0003\right)$ and inversely correlated with extracellular T14 levels in the cell culture supernatants $\left(r^{2}=-0.79 ; P=0.034\right)$. However, in the presence of NBP-14, a7 nAChR expression was reduced $(P=0.04)$ and the most migratory cells showed the largest reduction in expression. In conclusion, NBP-14-mediated antagonism of the a7 nAChR offers a novel therapeutic strategy with the potential to inhibit tumor cell migration.

\section{INTRODUCTION}

Nicotinic acetylcholine receptors (nAChRs) are ligand-gated ion channels expressed in the cell membrane of a wide range of mammalian cells, including cancer cells [1]. Recent findings suggest that nAChRs not only mediate diverse functions in the brain, such as learning, memory, addiction and general plasticity [2] but may also contribute to the development and progression of a diverse array of cancers [3]. Binding of acetylcholine to the $\alpha$ subunits of $n A C h R s$ results in a conformational change in the receptor allowing ions to flow from the extracellular space into the cell [4]. The influx of the cations causes membrane depolarization which in turn triggers the opening of voltage-activated calcium channels, leading to an additional influx of calcium. This promotes a host of diverse responses, including the release of neurotransmitters and angiogenic, neurotrophic and growth factors. In addition, the increased intracellular cation concentrations may trigger the direct stimulation of intracellular signaling cascades that are involved in the regulation of cell proliferation, apoptosis, migration and differentiation [5].

Over the last 45 years [6] a link between cancer cell proliferation and acetycholinesterase (AChE) has become increasingly well established. In addition to its conventional enzymatic role in hydrolyzing acetylcholine, AChE could have an effect in cancer, independent of cholinergic transmission, that involves a variety of potential functions such as cell adhesion, differentiation, 
regulation of apoptosis and proliferation [7]. One potential clue for these novel actions of AChE is the shift in patterns of oligomerization characterised during metastases, where the typical tetramer of four catalytic subunits disappears in favour of an increased number of dimers and monomers [8]. A possible explanation for this shift could be that the disulphide bond necessary for oligomerization is no longer intact, due to the cleavage of a constituent 14mer peptide (T14) at the C-terminal of AChE [9], rendering it available to act as a signaling molecule.

In a previous study, we showed that T14 had a selective action on the strongly metastatic breast cancer cell line MDA-MB-231 via the $\alpha 7 \mathrm{nAChR}$. In contrast, these effects were not evident on the less metastatic cell line MCF-7 [10]. Accordingly, the initial aim of this study was to investigate whether endogenous $\alpha 7 \mathrm{nAChR}$ and $\mathrm{T} 14$ could be detected in a range of cancer cell lines and primary malignant and non-malignant cells. Furthermore, since recent observations have indicated that the action of the AChE-peptide T14 on brain tissue can be blocked by a cyclized variant (NBP-14) [11], we characterized the effects of this cyclized variant in a range of human cancer cell lines as well as primary chronic lymphocytic leukemia cells and normal B-cells.

Here we show for the first time that the $\alpha 7 \mathrm{nAChR}$ plays a key role in modulating tumor cell migration. Moreover, there was a positive correlation between intracellular T14 and $\alpha 7 \mathrm{nAChR}$ expression and an inverse correlation between extracellular T14 derived from the culture media and $\alpha 7 \mathrm{nAChR}$ expression. We further demonstrate that exposure to the $\alpha 7 \mathrm{nAChR}$ antagonist and cyclized variant of T14, NBP-14, results in reduced tumor cell migration without any significant loss in cell viability. Furthermore, NBP-14 appears to preferentially effect tumor cell migration as normal B-cells were less sensitive to this cyclic peptide. The combination of these characteristics suggests that targeting the $\alpha 7 \mathrm{nAChR}$ may be a promising therapeutic strategy particularly in cancers with a strong metastatic potential.

\section{RESULTS}

\section{Intracellular and extracellular T14 expression correlate with $\alpha 7 \mathrm{nAChR}$ expression}

Our first goal was to determine whether we could detect the T14 peptide in samples collected from each of the cell lines and primary cells. We performed Western blot analysis for T14 and $\alpha 7 \mathrm{nAChR}$ on cell lysates and aliquots of the cell culture media. The $\alpha 7 \mathrm{nAChR}$ was not detectable in the cell culture media (data not shown) but was consistently detected in the cell lysates (Figure 1A). a7 nAChR expression levels were normalized to a GAPDH loading control revealing differential expression of $\alpha 7 \mathrm{nAChR}$ in the various cell lines. In contrast, T14 was detectable in both the cell lysates (Figure 1B) and the cell culture media (Figure 1C). It is worthy of note that T14 was also detected in cell-free media containing $10 \%$ fetal calf serum but this could not explain the quantitatively different T14 levels found in the cell lysates and cell culture supernatants. As previously described, T14 was detected as aggregates rather than as a monomeric form [12] thereby explaining the higher than expected molecular weight of the Western blot band. Furthermore, there was a strong positive correlation between intracellular $\mathrm{T} 14$ and $\alpha 7 \mathrm{nAChR}$ expression (Figure 1D; $r^{2}=0.91$ ) and an inverse correlation between extracellular T14 derived from the culture media and $\alpha 7 \mathrm{nAChR}$ expression (Figure 1E; $r^{2}=-0.79$ ).

\section{NBP-14 demonstrates low cytotoxic and only modest anti-proliferative effects}

All of the primary cells and cell lines used in this study were shown to express varying levels of the $\alpha 7 \mathrm{nAChR}$ (Figure 2A). Treatment with the $\alpha 7 \mathrm{nAChR}$ antagonist NBP-14 showed modest apoptotic effects in all of the cell lines tested at concentrations $>0.1 \mu \mathrm{M}$ (Figure 2B). Comparison of the apoptotic effects of NBP-14, T15 and T30 in each of the cell lines are shown in Supplementary Figure 1. Although MCF7 cells showed significantly increased sensitivity to NBP-14, these cells were equally sensitive to the effects of a control peptide (T15) consisting of the inert C-terminal 15 amino acid residues of the neuro-active peptide (T30) suggesting that these effects were non-specific (Figure 2C). Furthermore, NBP-14 was essentially non-toxic in normal B-cells at the concentrations tested and was significantly more toxic in malignant B-cells derived from chronic lymphocytic leukemia (CLL) patients (Figure 2D; $P<0.001)$. In terms of anti-proliferative activity, NBP-14 only showed evidence of cytostatic effects at concentrations of $>0.1 \mu \mathrm{M}$ (Figure 2E). Comparison of the anti-proliferative effects of NBP-14, T15 and T30 in each of the cell lines are shown in Supplementary Figure 2.

\section{NBP-14 preferentially inhibits the migration of primary cancer cells}

We next established the migratory potential of all of the primary cells and cell lines employed in this study using transwell assays. There was inherent variation in the propensity of these cells to migrate along a chemokine or serum gradient over a $24 \mathrm{~h}$ time period (Figure $3 \mathrm{~A}$ ). Interestingly, there was a positive correlation between $\alpha 7 \mathrm{nAChR}$ expression, as measured by flow cytometry, and baseline migration of the cell lines and primary cells investigated in this study although this did not reach statistical significance (Figure 3B; $r^{2}=0.31$ ). Noting these differences, we examined the effect on migration of the $\alpha 7 \mathrm{nAChR}$ inhibitory peptide NBP-14 over a range of concentrations. Figure 3C shows dose-response curves for effect of NBP-14 on the migration of the six cancer cell 
lines. Given that the cell lines (apart from MCF7 cells) showed a significant reduction in migration following exposure to $1 \mu \mathrm{M} \mathrm{NBP}-14$, we went on to determine the relative effects of $1 \mu \mathrm{M}$ of the inert $\mathrm{T} 15$ peptide, the T30 peptide containing the 14 amino acid sequence of T14 and the cyclized NBP-14 peptide in primary CLL cells, normal B-cells $(n=5)$ and each of the cell lines. Samples derived from ten CLL patients showed inherent

A alpha-7 nicotinic receptor in cell lysates

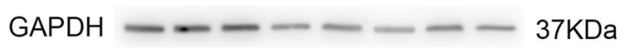

Alpha 7/GAPDH $\begin{array}{llllllll}1.8 & 1.6 & 1.8 & 3.2 & 2.8 & 3.5 & 3.2 & 3.8\end{array}$

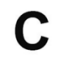

\section{T14 in cell culture media}

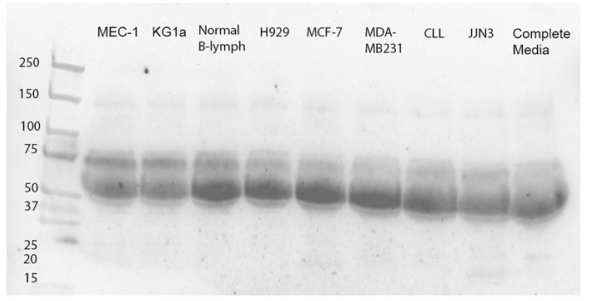

T14 -Complete Media/ $8.0 \quad 5.17 .0 \quad-2.1 \quad 1.5 \quad 1.7 \quad 1.6-1.5$ Total Protein

(all $10^{-2}$ )

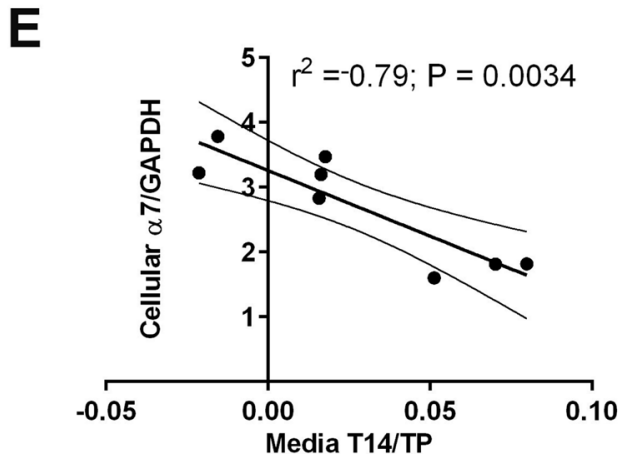

differences in migration (Figure 3D) but all showed a significant reduction in migration when cultured in the presence of $1 \mu \mathrm{M}$ NBP-14. In contrast, culture with T15 and $\mathrm{T} 30$ had no significant effect. The co-administration of T30 and NBP-14 had no significant effect beyond that achieved with NBP-14 alone. Normal B-cells also showed a significant reduction migration following exposure to NBP-14 (Figure 3E). However, despite manifesting similar

B

\section{T14 in cell lysates}

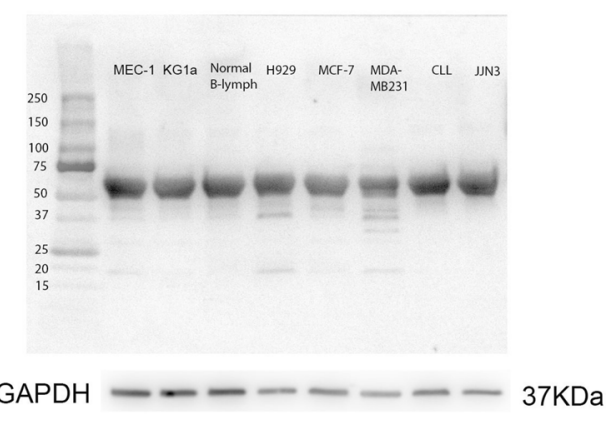

T14/GAPDH $\begin{array}{llllllll}1.8 & 1.6 & 1.8 & 3.0 & 2.1 & 3.0 & 3.0 & 3.1\end{array}$

\section{D}

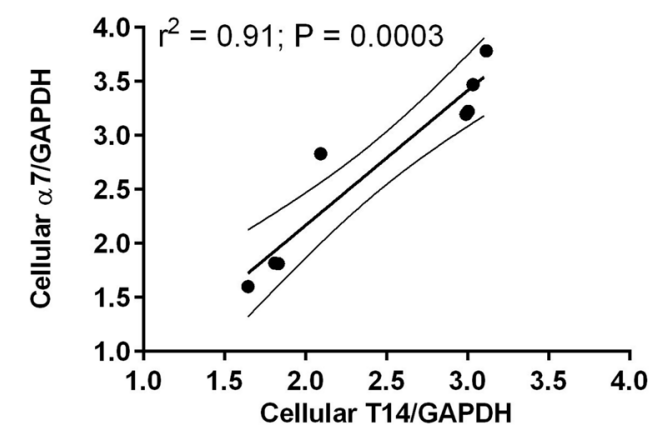

Figure 1: (A) $\alpha 7 \mathrm{nAChR}$ expression was assessed in whole cell lysates $\left(1 \times 10^{6}\right.$ cells $)$ from each cell type using Western blotting. Protein bands were quantified using Image J and the $\alpha 7 \mathrm{nAChR}$ expression was normalized to GAPDH in order to determine the relative expression of $\alpha 7 \mathrm{nAChR}$ in the cell lines and primary cells under investigation. (B) T14 levels in cell lysates were quantified in the same way and (C) the level of T14 present in complete media was subtracted from cell culture media derived from each of cell lines and primary cells, before normalising to total protein using Blot FastStain. (D) $\alpha 7 \mathrm{nAChR}$ positively correlated with intracellular T14 expression $\left(r^{2}=0.91\right.$, $P=0.0003)$ and $(\mathbf{E})$ negatively correlated with the levels of T14 detected in the culture media $\left(r^{2}=-0.79, P=0.0034\right)$. Graphs display the lines of best fit together with the $95 \%$ confidence intervals. 
A

MCF-7

MDA-MB-231
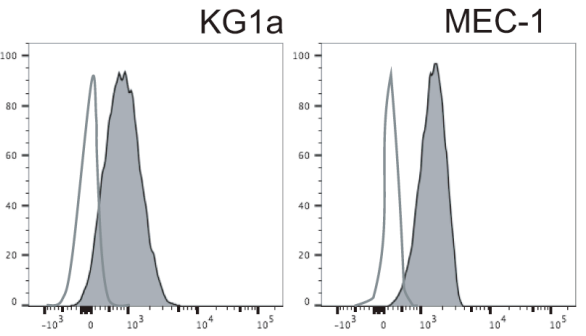

H929

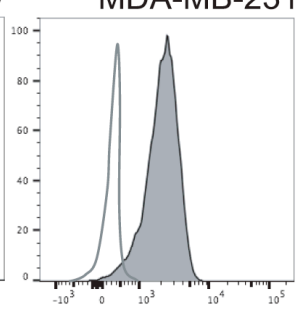

JJN3

Primary CLL
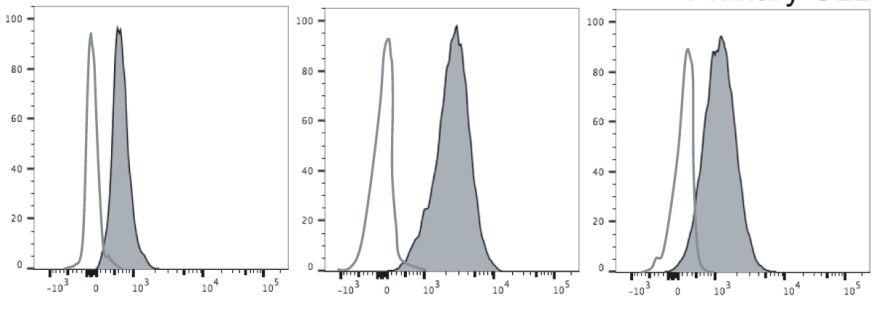

Normal B-cells

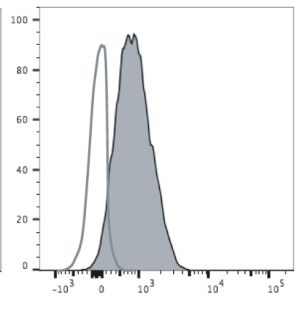

alpha-7 nicotinic receptor expression

B

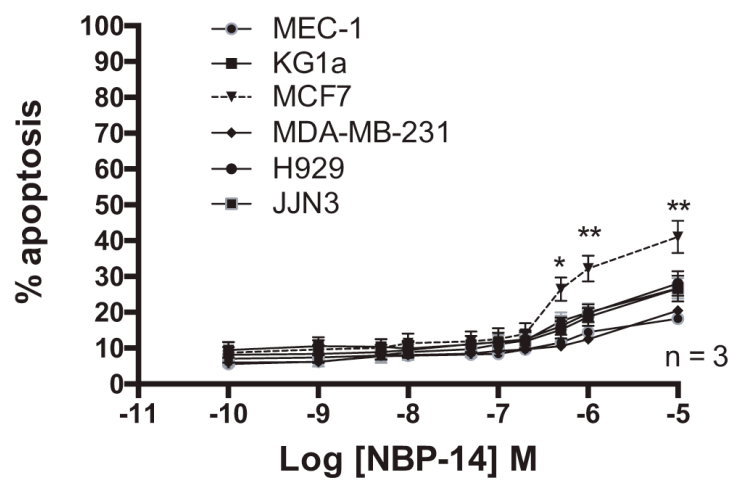

D

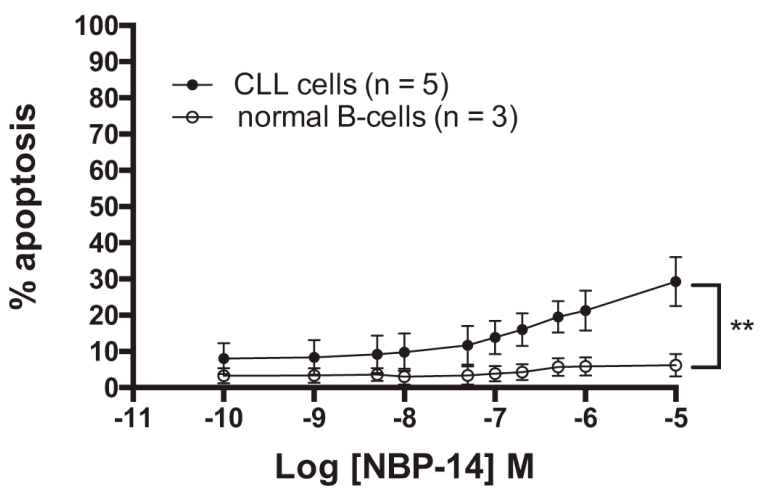

C

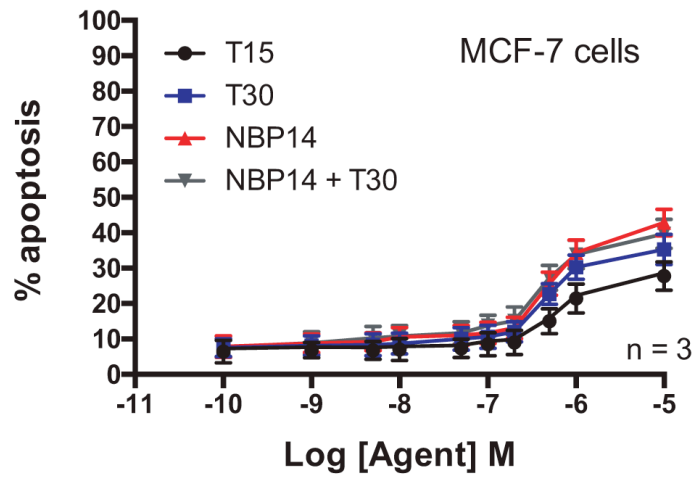

E

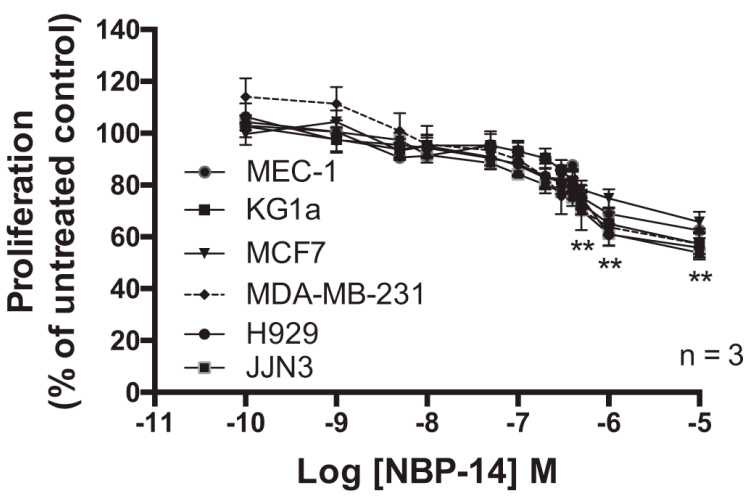

Figure 2: (A) Comparison of $\alpha 7 \mathrm{nAChR}$ expression on the surface of the cancer cell lines and primary cells used in the study. In each case cells not labelled with antibody were analyzed to determine the level of autofluorescence (open histograms). (B) Cytotoxic doseresponse curves were generated from flow cytometric analysis using Annexin V and propidum iodide labeling of each of the cancer cell lines following exposure to increasing concentrations of NBP-14 for $72 \mathrm{~h}$. (C) Comparison of the apoptotic effects of the cyclized peptide (NBP-14), the inert peptide T15, the T30 peptide containing the T14 active peptide amino acid sequence and the combination of NBP-14 and T30 in MCF-7 breast cancer cells. (D) The cytotoxic effect of NBP-14 on primary CLL cells $(n=5)$ and normal B-cells $(n=3)$. $(\mathbf{E})$ $\mathrm{NBP}-14$ induced a dose-dependent decrease in proliferation in all of the cell lines tested. All data are presented as mean $( \pm \mathrm{SD})$. $* P<0.05$ and $* * P<0.001$. 
levels of basal migration to leukemic CLL cells $(P=0.4)$, normal B-cells were significantly less sensitive to the effects of NBP-14 when compared with malignant B-cells derived from CLL patients (Figure $3 \mathrm{~F} ; P=0.0002$ ). It is possible that this may be attributable to the lower levels of $\alpha 7 \mathrm{nAChR}$ expressed on normal B-cells when compared to primary CLL cells.

\section{NBP-14 inhibits the migration of diverse cancer cell lines}

Having established that NBP-14 had preferential anti-migratory effects on primary cancer cells, we went on to investigate the effects of NBP-14 on breast cancer, multiple myeloma, acute myeloid leukemia and CLL cell lines (Figure 4A-4F). With the exception of MCF-7 cells, all of the other cell lines showed a significant reduction in migration when treated with $1 \mu \mathrm{M}$ NBP-14. As was the case with primary CLL cells, the co-administration of T30 and NBP-14 had no additional effect on migration when compared with NBP-14 alone. It is worthy of note that KG1a cells do not express CXCR4, the receptor for the chemokine CXCL12, and so in this case migration was promoted by the addition of $20 \%$ FCS into the basolateral chamber of the transwells. NBP-14 was able to inhibit the migration of KGla cells under these conditions suggesting that the mechanism of inhibition is independent of chemokine receptor expression.

\section{Basal migration predicts for response to NBP-14}

Having established that the different cell lines and primary cells had inherent differences in basal migration, we examined whether this was predictive of response to NBP-14. Figure 5A shows the cell lines ranked according to the percentage decrease in migration induced by $1 \mu \mathrm{M}$ NBP-14. There was a clear correlation between basal migration and the percentage decrease in migration induced by NBP-14 (Figure 5B; $r^{2}=0.45 P=0.06$ ). Furthermore, when the normal B-cells were excluded from the analysis the correlation was even stronger suggesting that tumor cells may be more reliant on T14 signaling in order to migrate (Figure $5 \mathrm{C} ; r^{2}=0.80 P=0.006$ ). Given that we had already established that there was variation in $\alpha 7 \mathrm{nAChR}$ expression between the cells under investigation, we set out to determine the effect of NBP-14 on $\alpha 7 \mathrm{nAChR}$ expression. Figure 5D shows that MDAMB-231 cells showed a marked reduction in $\alpha 7 \mathrm{nAChR}$ expression following treatment with NBP-14. In contrast, MCF-7 cells showed only a small reduction in $\alpha 7 \mathrm{nAChR}$ expression under the same conditions. When considering all of the cell types together, there was a significant reduction in $\alpha 7 \mathrm{nAChR}$ expression following exposure to NBP-14 (Figure 5E; $P=0.04$ ). Furthermore, there was a strong positive correlation between the NBP-14-mediated decrease in migration and the percentage decrease in $\alpha 7 \mathrm{nAChR}$ expression (Figure 5F; $r^{2}=0.83, P=0.0016$ ). Although we only analyzed $\alpha 7 \mathrm{nAChR}$ expression in a small cohort of primary CLL patients $(n=6)$, there was heterogeneity in basal $\alpha 7 \mathrm{nAChR}$ expression and also the degree of $\alpha 7 \mathrm{nAChR}$ inhibition induced by NBP-14 (Supplementary Figure 3).

\section{DISCUSSION}

Cancer recurrence at secondary locations, often years after effective treatment of the primary tumor, accounts for most of the mortality associated with solid tumors [13]. Furthermore, the ability of leukemia cells to infiltrate tissues and take advantage of pro-survival microenvironments likely contributes to their chemoresistance. Therefore, understanding the cellular processes that promote metastasis and clinical relapse is of critical importance. In this regard, the identification of novel therapeutic targets is of particular interest because they are less likely to be subject to the common drug resistance mechanisms encountered following treatment with chemotherapy or immunotherapy [14].

$\mathrm{AChE}$ has been implicated in tumorogenesis for almost 50 years [6], in many cases in a capacity not linked to its conventional enzymatic role in cholinergic transmission [7]. Of particular interest is the observation that in metastatic mode, there is a shift from a high level of tetramers, to a higher proportion of dimers and monomers [8]. One interpretation of this change in oligomerization pattern is that during metastasis there is an enhanced availability of free C-terminal peptide resulting in significantly increased levels of endogenous T14. A role for $\mathrm{T} 14$ in metastases was previously inferred by the effects of exogenous T14 on the highly metastatic breast cancer cell line MDA-MB-231 [10]. Recently a cyclized peptide, NBP-14, has been shown to antagonize the effects of T14 in brain tissue [15]. Using this new agent, we set out to assess whether $\alpha 7 \mathrm{nAChR}$ antagonism could provide a novel therapeutic target in human cancers. In particular, we wanted to establish whether this peptide could impact upon the migratory capacity of a range of tumor cell lines and primary CLL cells. We compared our findings with those obtained using normal B-cells.

As a first step, we confirmed that all of the cells under evaluation in this study expressed the $\alpha 7 \mathrm{nAChR}$. We noted variation in receptor expression but this was not associated with the modest cytotoxic or anti-proliferative effects induced by NBP-14. It was of considerable interest that we were able to detect the active T14 peptide in both cellular extracts and the media that they were cultured in. Indeed, we were able to demonstrate a strong positive correlation between the intracellular levels of T14 and the expression of the $\alpha 7 \mathrm{nAChR}\left(r^{2}=0.91\right)$. Furthermore, there was an inverse correlation between T14 detected in the culture media and expression of the $\alpha 7 \mathrm{nAChR}\left(r^{2}=-0.79\right)$. 
A

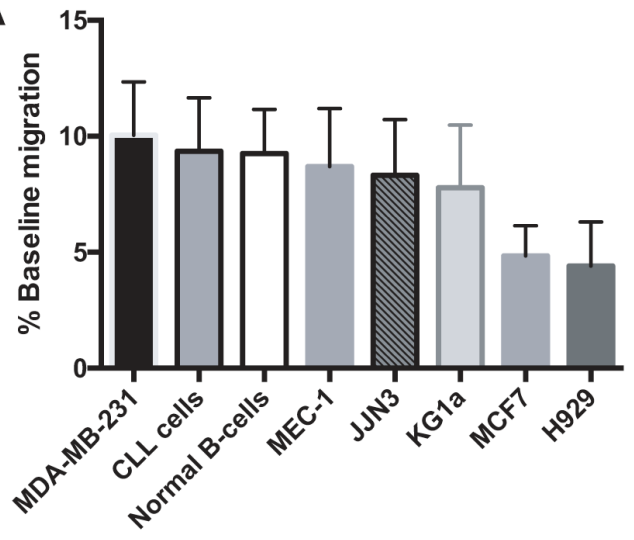

C

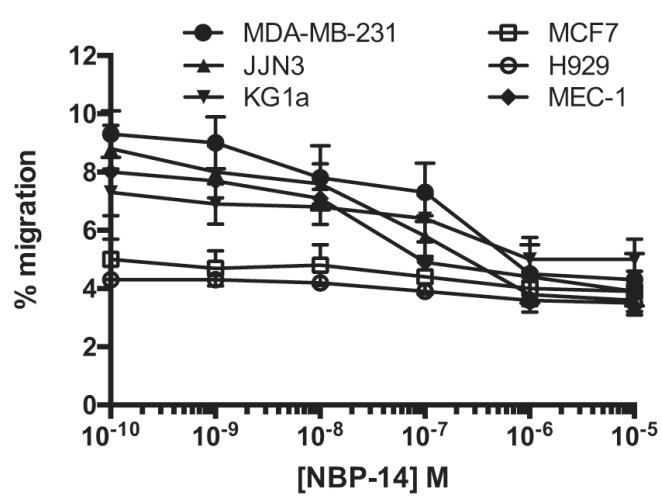

E

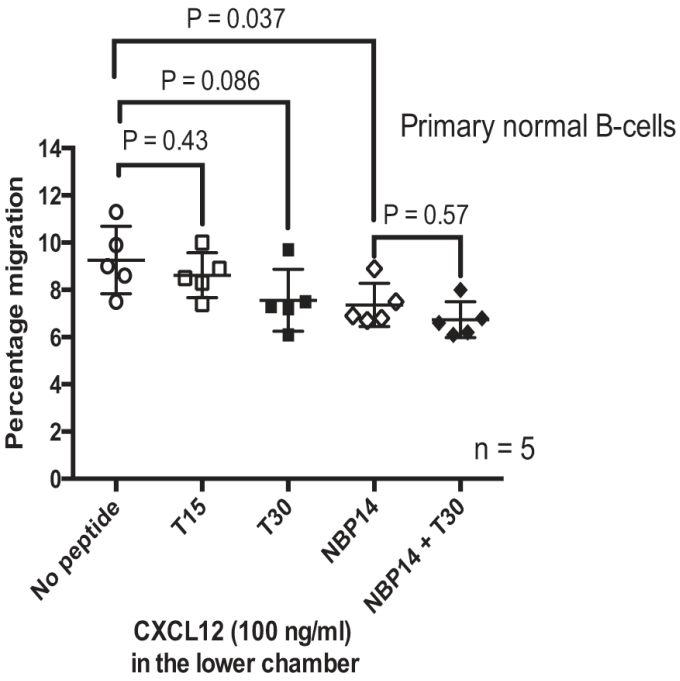

B

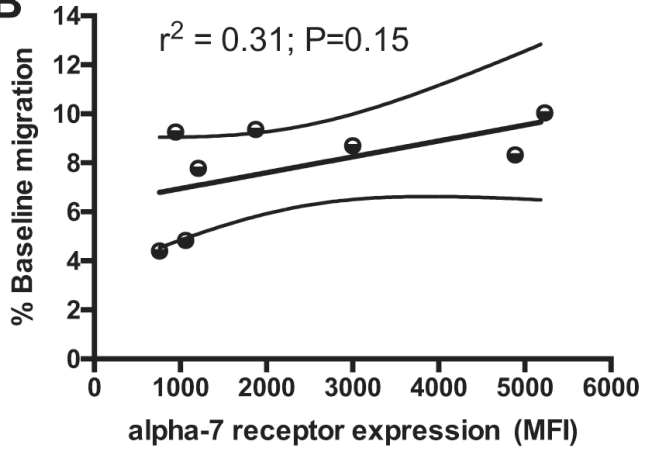

D

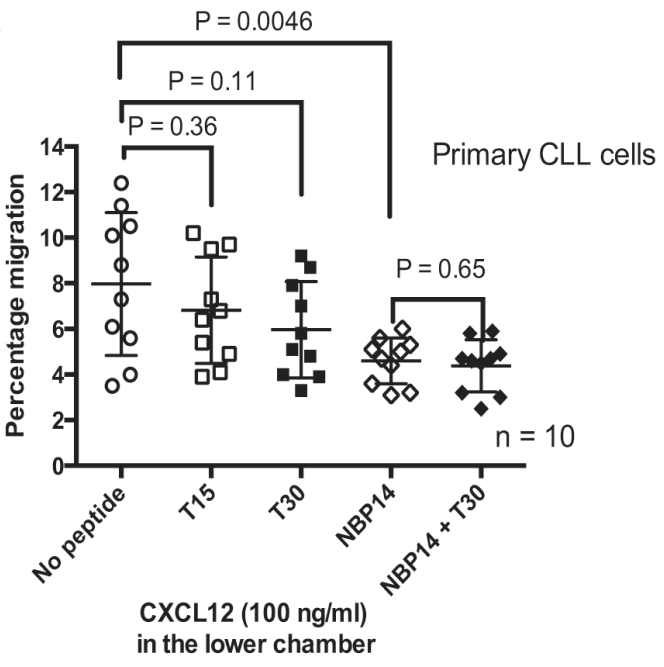

F

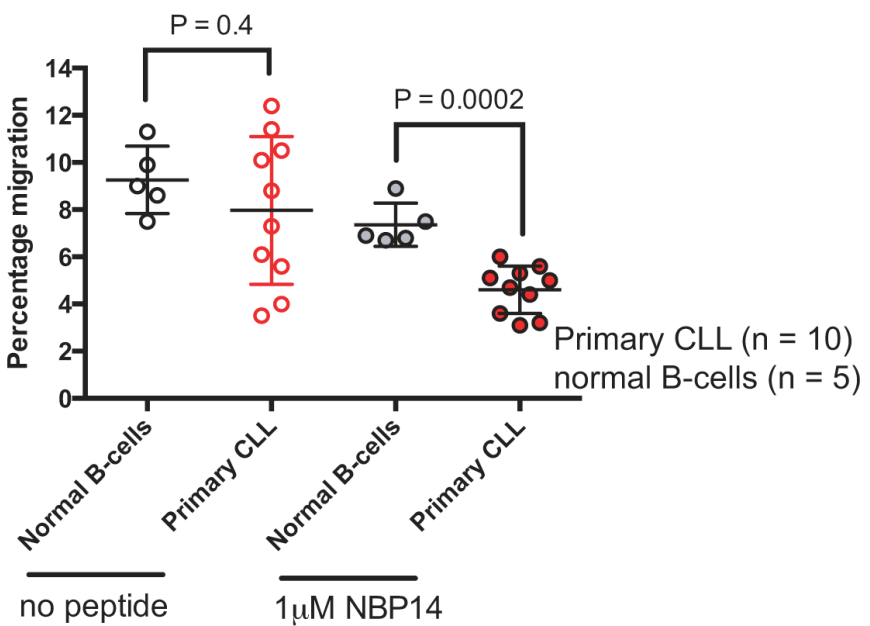

Figure 3: (A) Cell migration in transwells was quantified over after $24 \mathrm{~h}$ and the mean baseline percentage migration for each of the cell lines and primary cells were arranged in descending order. (B) There was a positive correlation $\left(r^{2}=0.31\right)$ between percentage baseline migration and $\alpha 7 \mathrm{nAChR}$ expression. (C) The inhibitory effect on migration induced by NBP-14 was dose-dependent up to $1 \mathrm{mM}$ for each of the six cancer cell lines tested. Comparison of the anti-migratory responses induced by $1 \mathrm{mM}$ of the T15, T30 and NBP-14 peptides in (D) primary CLL cells and (E) primary normal B-cells. (F) Although there was no statistical difference in baseline migration between normal B-cells and CLL B-cells, 1 mM NBP-14 preferentially inhibited the migration of primary CLL cells when compared with normal B-cells. 
A

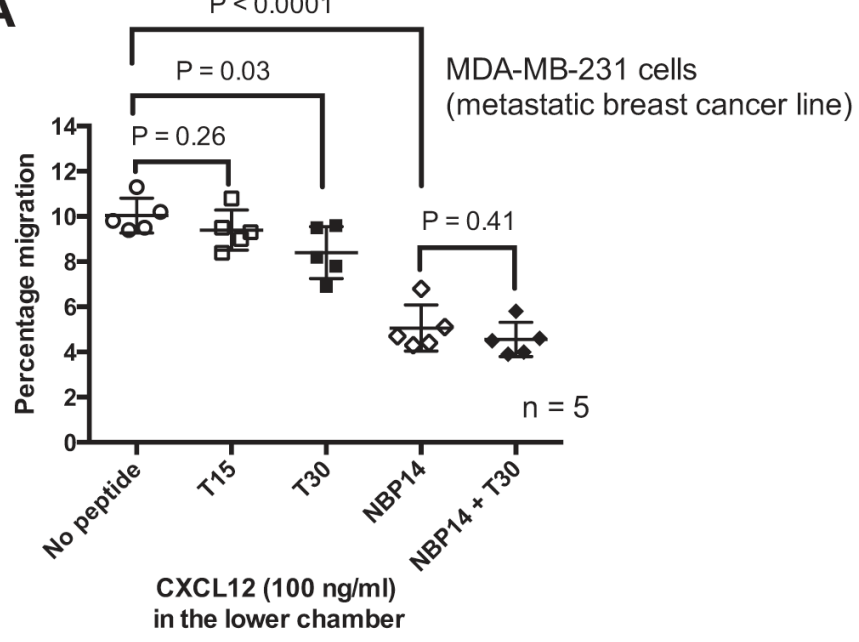

C

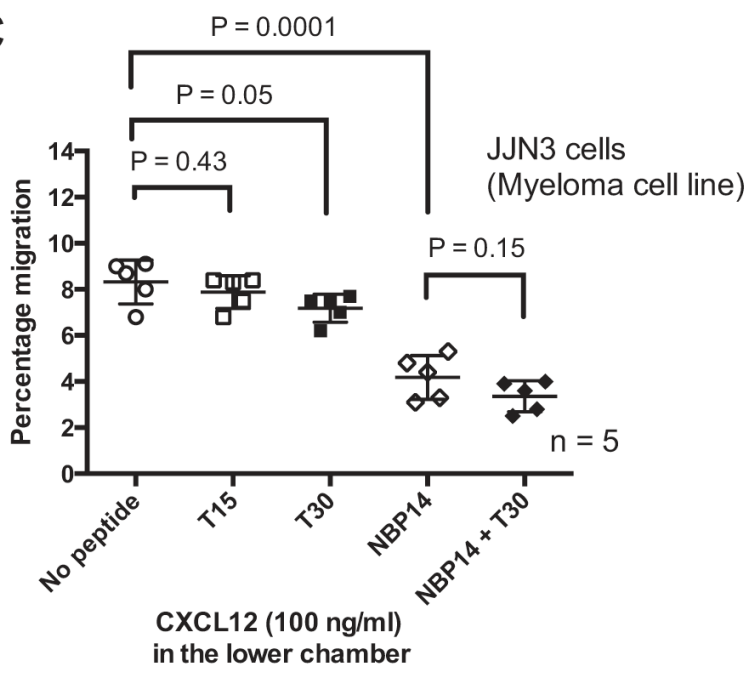

E

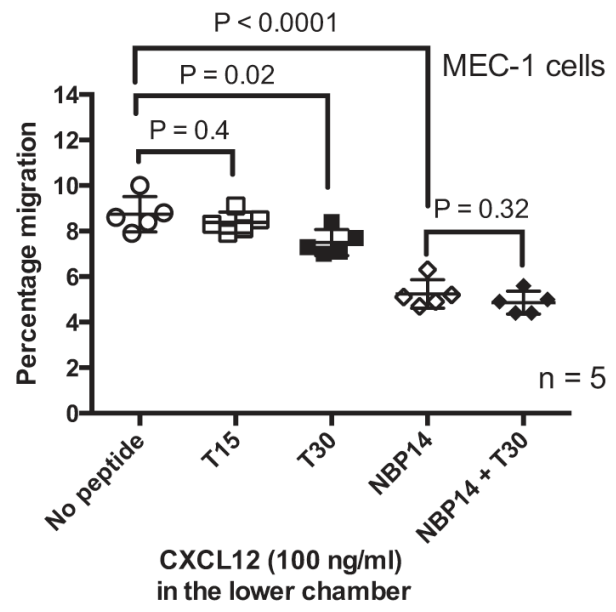

B

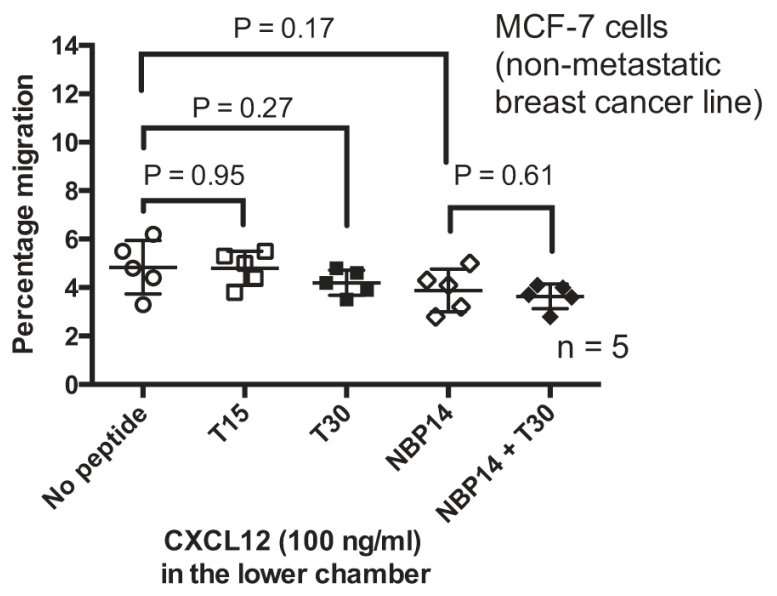

D

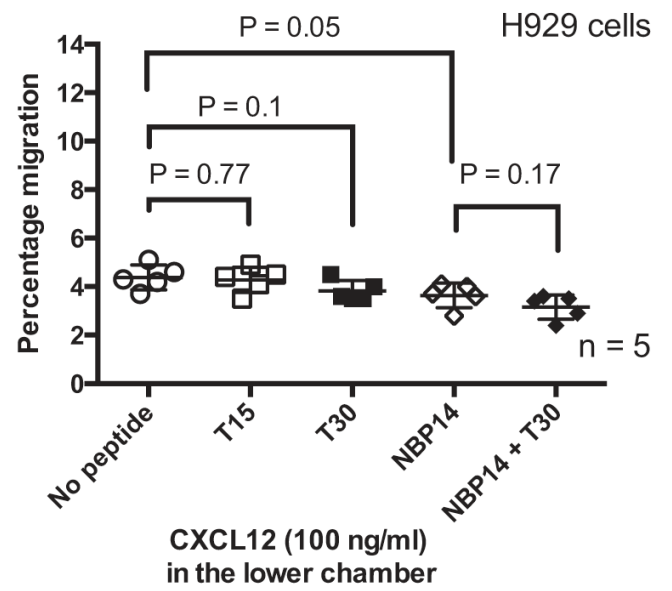

$\mathbf{F}$

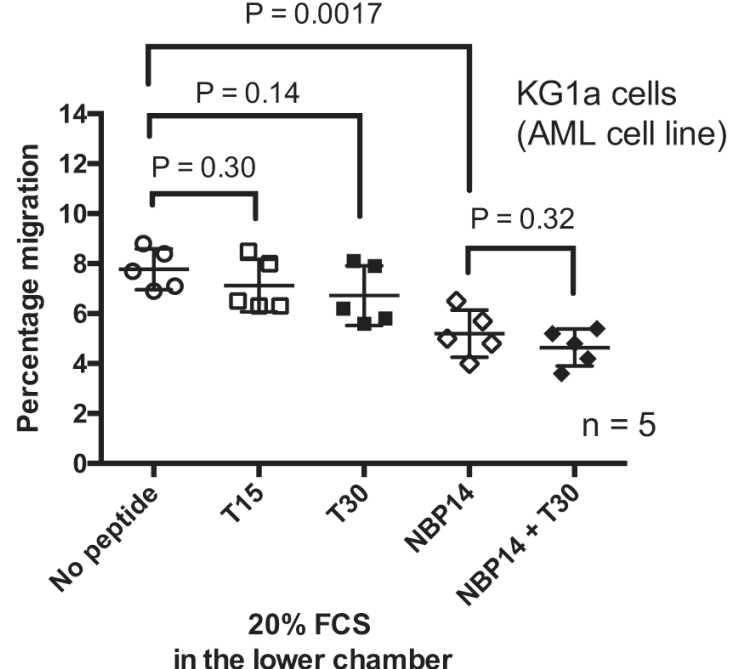

Figure 4: The anti-migratory responses induced by the T15, T30 and NBP-14 peptides were assessed in transwell experiments conducted over $24 \mathrm{~h}$ in (A) MDA-MB-231 cells (B) MCF-7 cells (C) JJN3 cells (D) H929 cells (E) MEC-1 cells and (F) KG1a cells. All of the cell lines, apart from MCF7 cells, showed a significant reduction in migration following co-incubation with $1 \mathrm{mM}$ NBP-14. The T30 peptide had more variable effects on the cell lines but in every case the inert T15 peptide had no significant impact on the migration of the cells. All data are presented as mean $( \pm \mathrm{SD})$ of five independent experiments. 

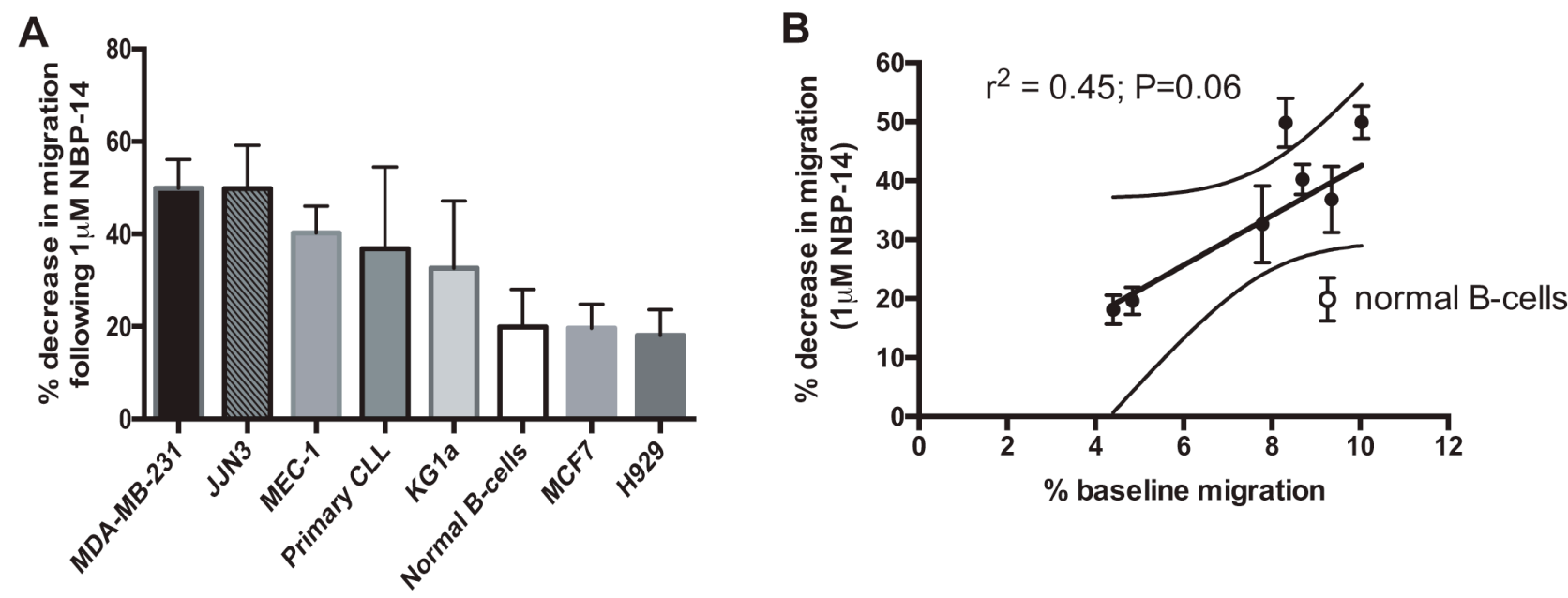

C
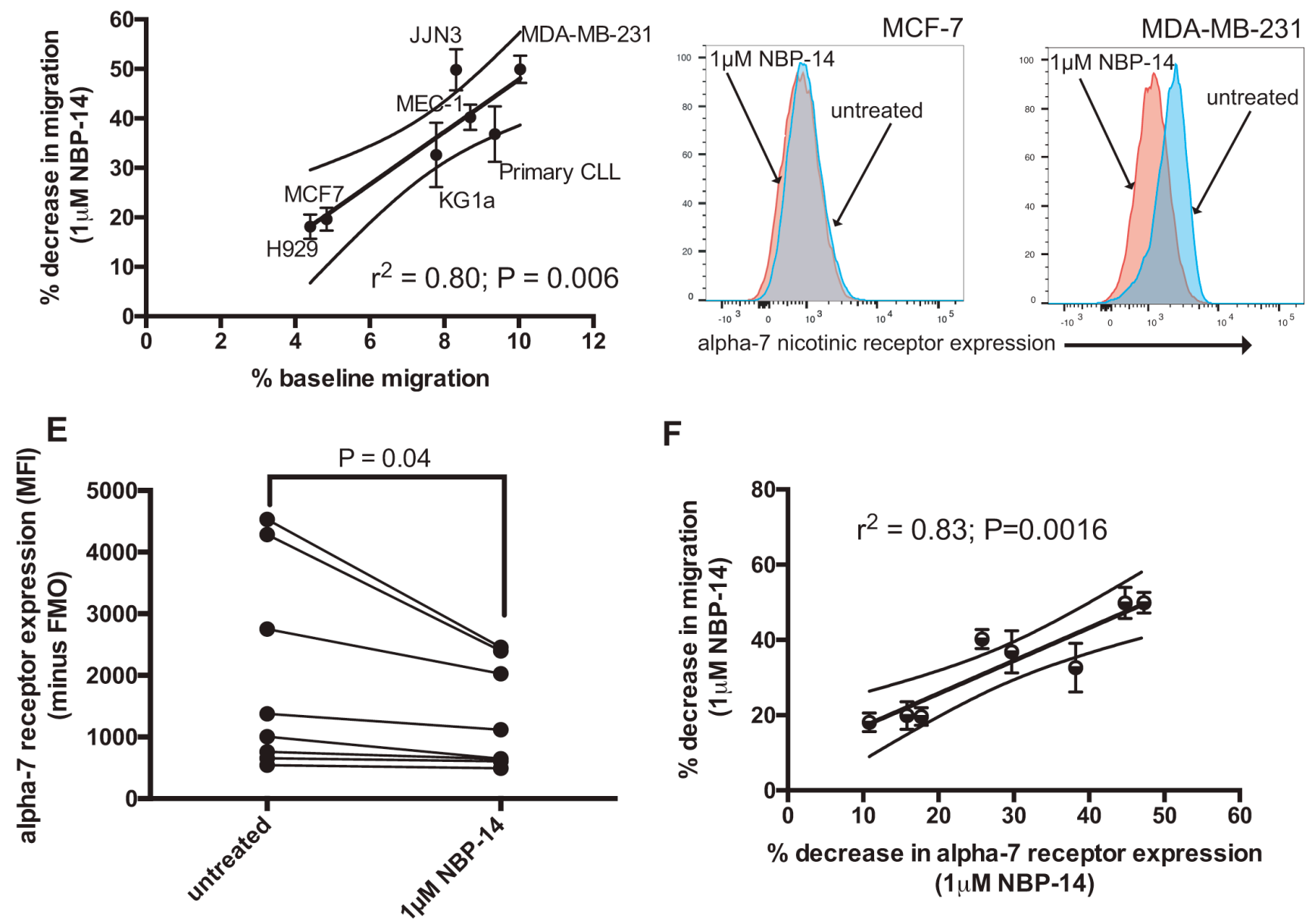

F

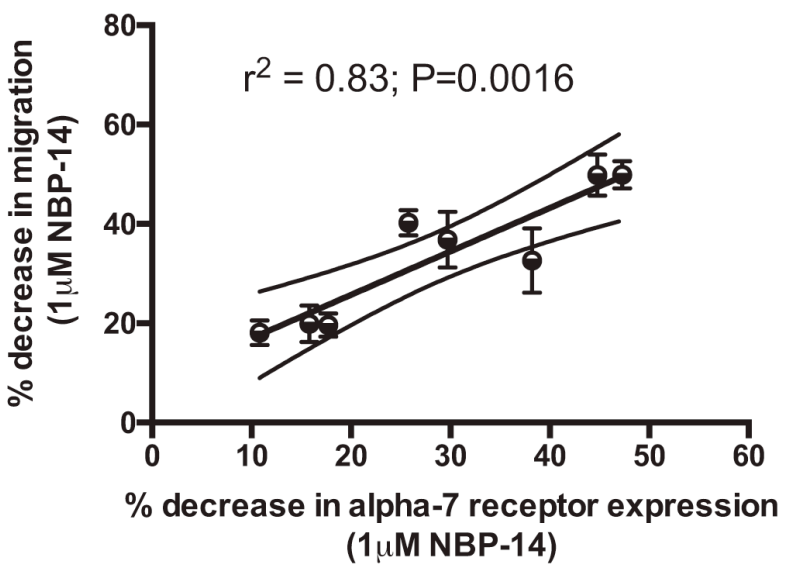

Figure 5: The change in migration induced by $24 \mathrm{~h}$ exposure to NBP-14 was calculated for each cell type. (A) shows the mean percentage decrease in migration induced by $1 \mathrm{mM}$ NBP-14 for each of the cell lines and primary cells arranged in descending order. (B) We next plotted the change in migration induced by NBP-14 against the percentage baseline migration. These parameters were positively correlated $\left(r^{2}=0.45\right)$ and $(\mathbf{C})$ was strengthened when the normal B-cell data was excluded $\left(r^{2}=0.80\right)$. (D) In parallel experiments, we assessed the effect of exposure to $1 \mathrm{mM} \mathrm{NBP-14}$ on the surface expression of the $\alpha 7 \mathrm{nAChR}$ on MCF7 and MDA-MB-231 cells. NBP-14 caused a reduction in $\alpha 7 \mathrm{nAChR}$ expression on both cell lines; this was most apparent on MDA-MB-231 cells, which manifest higher constitutive expression of the receptor. (E) Analysis of the other cell lines and primary cells confirmed that $\alpha 7 \mathrm{nAChR}$ expression was significantly reduced following exposure to $1 \mu \mathrm{M} \mathrm{NBP}-14$ for $72 \mathrm{~h}$. (F) Furthermore, the decrease in migration induced by NBP-14 strongly correlated with the decrease in $\alpha 7 \mathrm{nAChR}$ expression $\left(r^{2}=0.83\right)$. 
Despite its lack of potency as a cytotoxic/cytostatic agent, NBP-14 was able to preferentially inhibit the migration of tumor cells when compared to normal B-cells. Moreover, the exogenous, linear peptide T30, when applied alone or in combination with NBP-14 had no significant effect on migration when compared with NBP-14 alone in any of the cell lines and primary cells evaluated. This could be due to a ceiling effect of endogenous T14 preventing any further effects of exogenous T30. This concept was supported by the observation that the most migratory cells manifested the highest levels of $\alpha 7 \mathrm{nAChR}$, the lowest levels of extracellular T14 but the highest levels of intracellular T14. This implies that these cells are capable of scavenging T14 from their microenvironment resulting in optimal intracellular signaling. Exposure to the cyclized variant, NBP-14, caused a significant reduction in surface expression of $\alpha 7 \mathrm{nAChR}$ presenting a plausible mechanism for blocking the actions of endogenous T14.

Primary CLL cells showed baseline heterogeneity in both their $\alpha 7 \mathrm{nAChR}$ expression and their migratory capacity. However, NBP-14 was able to significantly reduce migration in all of the primary tumor samples tested. It is of particular interest that although normal B-cells showed a similar level of baseline migration to leukemic B-cells, they were significantly less sensitive to the anti-migratory effects of NBP-14 $(P=0.0002)$. This implies normal B-cells are not as dependent on the effects of $\alpha 7 \mathrm{nAChR}$ signaling as their malignant counter-parts presenting the potential for a positive therapeutic index when inhibiting this target. A rationale for these observed differential effects is that normal B-cells expressed less $\alpha 7 \mathrm{nAChR}$ when compared with leukemic B-cells. This concept was further supported by the finding that there was a strong positive correlation between the reduction in $\alpha 7 \mathrm{nAChR}$ expression and the reduction in migration induced by NBP-14 in all of the cell lines and primary cells evaluated.

In conclusion, the findings of this study support the concept that antagonizing $\alpha 7 \mathrm{nAChR}$ signaling in human cancers represents a promising therapeutic strategy. Although the precise downstream consequences of $\alpha 7 \mathrm{nAChR}$ signaling remain unresolved, it is clear that the molecular targets relate to migration rather than proliferation and survival. This raises the possibility of combining $\alpha 7 \mathrm{nAChR}$ antagonists, such as the novel agent NBP-14 described here, with other anti-cancer agents both in the frontline and relapsed/refractory settings.

\section{MATERIALS AND METHODS}

\section{Culture conditions for cancer cell lines, primary CLL cells and normal B-cells}

Primary chronic lymphocytic leukemia (CLL) cells were obtained from patients attending outpatients' clinics at the University Hospital of Wales with informed consent in accordance with the ethical approval granted by South East Wales Research Ethics Committee (02/4806). Normal B-cells were obtained from healthy volunteers again with informed consent. All of the cell lines were purchased from DSMZ and were used for these experiments within 6 months of purchase. In each case multiplex PCR of minisatellite markers revealed a unique DNA profile and were shown to be mycoplasma-free. All of the cell lines, the primary CLL cells and normal B-cells were maintained in RPMI medium supplemented with $100 \mathrm{units} / \mathrm{ml}$ penicillin, $100 \mu \mathrm{g} / \mathrm{ml}$ streptomycin and $10 \%$ fetal calf serum. In addition, $100 \mu \mathrm{M}$ of acetylcholine (Sigma) was added to the culture media to ensure that the availability of acetylcholine was not a limiting factor in these experiments. Cells were treated with a range of concentrations of peptide $(0-10 \mu \mathrm{M})$; all peptides (T15, T30 and NBP-14) were all synthesized by Genosphere Biotechnologies.

\section{Purification of CLL and normal B-cells}

Freshly isolated peripheral blood B-cells derived from CLL patients and healthy volunteers were isolated from whole blood by positive selection using CD19 microbeads on an AutoMACS Pro separator (Miltenyi Biotec.). Cell purity was subsequently checked using an Accuri C6 flow cytometer (BD Biosciences) after staining an aliquot of cells with a fluorescein isothiocyanate-labeled CD20 antibody (Biolegend). In every case $>94 \%$ of the cells analyzed were expressing CD20.

\section{Measurement of in vitro apoptosis}

Aliquots of each cell type $\left(1 \times 10^{6}\right.$ cells $)$ were cultured for $72 \mathrm{~h}$, harvested by centrifugation (300 $\mathrm{g}$ for 5 mins) and then resuspended in $195 \mu \mathrm{L}$ of calcium-rich buffer. Subsequently, $5 \mu \mathrm{L}$ of Annexin V (eBiosciences) was added to the cell suspension and cells were incubated in the dark for 10 mins prior to washing. Cells were finally resuspended in $190 \mu \mathrm{L}$ of calcium-rich buffer together with $10 \mu \mathrm{L}$ of propidium iodide. Apoptosis was assessed by dual-color immunofluorescent flow cytometry using an Accuri C6 flow cytometer and data were analyzed using CFlow software (BD Biosciences).

\section{Measurement of in vitro proliferation}

Cultured cells were harvested by centrifugation and were then counted using a Vi-Cell XR cell viability counter (Beckman Coulter). The number of viable cells in each culture was then expressed as a percentage of the viable cells in the control cultures (no peptide).

\section{Western blotting}

Whole cell pellets $\left(1 \times 10^{6}\right.$ cells $)$ from eight cancer cell lines (MEC-1, KG1a, JJN3, H929, MCF-7, MDA- 
MB-231, primary CLL cells and normal B-cells) were solubilized in $1 \mathrm{x}$ Lysis Buffer (20 mM Tris Base, $137 \mathrm{mM}$ $\mathrm{NaCl}, 1 \%$ Tween-20, 2 mM EDTA) containing protease inhibitor cocktails (Phosphatase 1:1, PMSF 1:1, aprotinin $1: 1$ ) with a $17 \% \mathrm{v} / \mathrm{v}$ ratio. Subsequently, the mixture was triturated using a Polytron for 10 seconds and shaken at $4^{\circ} \mathrm{C}$ for $2 \mathrm{~h}$. Then, the samples were centrifuged at $13,000 \mathrm{~g}$ for 30 minutes at $4^{\circ} \mathrm{C}$ and the supernatants were taken and stored at $-80^{\circ} \mathrm{C}$. Protein concentrations were determined in the cell lysate samples above and their corresponding culture media samples using the Pierce ${ }^{\mathrm{TM}} 660 \mathrm{~nm}$ Protein Assay (Thermo Scientific, 22660). Subsequently, Western blot analysis was conducted on the samples using the previous established method [15]. The primary antibodies used were anti-T14 antibody $(1: 1000)$ [15], GAPDH (abcam, Ab181602, 1:1000) and anti- $\alpha 7$ nAChR (abcam, Ab10096, 1:1000). The secondary antibody used was goat anti-rabbit antibody conjugated to horseradish peroxidase (Abcam, ab6721, 1:5000). Protein bands derived from the cell lysates were quantified using Image J, measuring total optical intensity, and were subsequently normalized to the GAPDH loading control. In the case of the culture supernatants, T14 and $\alpha 7 \mathrm{nAChR}$ expression were normalized to total protein levels using Blot FastStain [16] to control for loading error.

\section{Flow cytometric analysis of Alpha-7 nicotinic receptor expression}

Aliquots of $1 \times 10^{6}$ cells from each cell line or primary cell sample were harvested by centrifugation, resuspended in $100 \mu \mathrm{L}$ staining buffer (PBS $+5 \%$ FCS) and then labeled with $10 \mu \mathrm{L}$ anti- $\alpha 7 \mathrm{nAChR}$ antibody directly conjugated with phycoerythrin (Santa Cruz Biotech., Sc-58607-PE). Cells were incubated with primary antibodies at $4^{\circ} \mathrm{C}$ for $20 \mathrm{~min}$ in the dark to prevent photobleaching. The cell suspension was then washed in $1 \mathrm{~mL}$ of staining buffer and prior to resuspension in $200 \mu \mathrm{L}$ of $1 \%$ paraformaldehyde solution. At least 10,000 cells were analyzed using an Accuri C6 flow cytometer (Becton Dickinson) and data were expressed as mean fluorescence intensity (MFI) values. In order to control for autofluorescence, the $\alpha 7 \mathrm{nAChR}$ MFI values were corrected by subtracting the FL2 MFI values derived from the analysis of unlabeled cells.

\section{Drugs and reagent}

Acetylthiocholine chloride was provided by SigmaAldrich, A5626. T30, T15 and NBP14 were synthesized by Genosphere Biotechnologies (France).

\section{Migration assays}

In vitro migration assays were performed by using $6.0 \mu \mathrm{m}$ pore size transwell migration plates (Costar, Corning). Aliquots $\left(1 \times 10^{6}\right.$ cells $)$ of each cell type in
$500 \mu \mathrm{l}$ of supplemented RPMI media were mixed with the appropriate concentration of peptide and were then added to the upper chamber of the transwell insert. $100 \mathrm{ng} / \mathrm{ml}$ of CXCL12 was added to the baso-lateral chamber for all the cell types tested apart from KG1a cells. KG1a cells do not express CXCR4 and so are unresponsive to CXCL12. Instead, media containing $20 \%$ fetal calf serum was added to the baso-lateral chamber in these experiments. The plates were incubated for $24 \mathrm{~h}$ at $37^{\circ} \mathrm{C}$ in $5 \% \mathrm{CO}_{2}$ in the presence of the peptides (T15, T30, NBP-14 and the combination of $\mathrm{T} 30+\mathrm{NBP}-14)$ at concentrations between $0.1 \mathrm{nM}$ and $10 \mu \mathrm{M}$. In addition, control cultures were carried out to which no peptide was added. Cells were subsequently harvested by centrifugation and were analyzed by flow cytometry using an Accuri C6 flow cytometer (BD). None of the conditions tested induced significant cell death in the cultures. Migration of CLL cells was determined by counting cells that migrated to the lower (baso-lateral) chamber of the transwell plate and then expressed as a percentage of the total number of cells initially added to the upper (apical) chamber.

\section{Statistical analysis}

The data obtained in these experiments were evaluated using the Wilcoxon signed rank test and correlation coefficients were calculated from least squares linear regression plots. $\mathrm{LD}_{50}$ values were calculated from line of best-fit analysis of the dose-response curves. All statistical analyses were performed using Graphpad Prism 6.0 software (Graphpad Software Inc., San Diego, CA).

\section{ACKNOWLEDGMENTS}

The work described in this paper is covered by patent applications: WO2016097753 A1 and WO2016156803 A1. The former covers the work on prevention of cancer metastasis by NBP14 while the latter focuses on the characterisation of the anti-T14 antibody.

\section{CONFLICTS OF INTEREST}

$\mathrm{CP}$ and $\mathrm{CF}$ acted as consultants for Neuro-Bio Ltd., HT, PM and SG-R are employees of Neuro-Bio Ltd., SG has shares in Neuro-Bio Ltd.

\section{Authors' contributions}

$\mathrm{CP}$ designed and performed experiments, analyzed data and revised the manuscript; HT performed experiments, analyzed data and revised the manuscript; PM performed experiments and analyzed data; SG-R analyzed data and revised the manuscript, $\mathrm{CF}$ designed experiments and revised the manuscript; SG conceived the overall original approach, designed experiments and wrote the manuscript. 


\section{REFERENCES}

1. Wessler I, Kirkpatrick CJ. Acetylcholine beyond neurons: The non-neuronal cholinergic system in humans. Br J of Pharmacol. 2009; 154:1558-1571. doi: 10.1038/ bjp.2008.185.

2. Nees F. The nicotinic cholinergic system function in the human brain. Neuropharmacol. 2015; 96:289-301. doi: 10.1016/j.neuropharm.2014.10.021.

3. Schuller HM. Is cancer triggered by altered signalling of nicotinic acetylcholine receptors?. Nat Rev Cancer. 2009; 9:195-205. doi: 10.1038/nrc2590.

4. Le Novere N, Changeux JP. Molecular evolution of the nicotinic acetylcholine receptor: An example of multigene family in excitable cells. J Mol Evol. 1995; 40:155-172. doi: 10.1007/bf00167110.

5. Roderick HL, Cook SJ. $\mathrm{Ca}^{2+}$ signalling checkpoints in cancer: Remodelling $\mathrm{ca}^{2+}$ for cancer cell proliferation and survival. Nat Rev Cancer. 2008; 8:361-375. doi: 10.1038/ $\operatorname{nrc2374.~}$

6. Blume A, Gilbert F, Wilson S, Farber J, Rosenberg R, Nirenberg M. Regulation of acetylcholinesterase in neuroblastoma cells. Proc Natl Acad Sci USA. 1970; 67:786-92.

7. Xi HJ, Wu RP, Liu JJ, Zhang LJ, Li ZS. Role of acetylcholinesterase in lung cancer. Thorac. Cancer. 2015; 6:390-398. doi: 10.1111/1759-7714.12249.

8. Vidal CJ. Expression of cholinesterases in brain and non-brain tumours. Chem Bio Interact. 2005; 157-158: 227-232. doi: 10.1016/j.cbi.2005.10.035.

9. Greenfield S. Discovering and targeting the basic mechanism of neurodegeneration: The role of peptides from the c-terminus of acetylcholinesterase. Chem Bio Interact. 2013; 203:543-546. doi: 10.1016/j.cbi.2013.03.015.
10. Onganer PU, Djamgoz MBA, Whyte K, Greenfield SA. An acetylcholinesterase-derived peptide inhibits endocytic membrane activity in a human metastatic breast cancer cell line. Biochim. Biophys Acta - Gen Subjects. 2006; 1760:415-420. doi: 10.1016/j.bbagen.2005.12.016.

11. Badin AS, Morrill P, Devonshire IM, Greenfield SA. (II) physiological profiling of an endogenous peptide in the basal forebrain: Age-related bioactivity and blockade with a novel modulator. Neuropharmacol. 2016; 105:47-60. doi: 10.1016/j.neuropharm.2016.01.012.

12. Cottingham M, Hollinshead, Vaux D. Amyloid fibril formation by a synthetic peptide from a region of human acetylcholinesterase that is homologous to the Alzheimer's amyloid-beta peptide. Biochem. 2002; 41:13539-47. doi: 10.1021/bi0260334.

13. Coghlin C, Murray GI. Current and emerging concepts in tumour metastasis. J Pathol. 2010; 222:1-15. doi: 10.1002/ path.2727.

14. Kachalaki S, Ebrahimi M, Mohamed Khosroshahi L, Mohammadinejad S, Baradaran B. Cancer chemoresistance; biochemical and molecular aspects: A brief overview. Eur J Pharm Sci. 2016; 89;20-30. doi: 10.1016/j.ejps.2016.03.025.

15. Garcia-Ratés S, Morrill P, Tu H, Pottiez G, Badin AS, Tormo-Garcia C, Heffner C, Coen CW, Greenfield SA. (I) pharmacological profiling of a novel modulator of the $\alpha 7$ nicotinic receptor: Blockade of a toxic acetylcholinesterasederived peptide increased in Alzheimer brains. Neuropharmacol. 2016; 105:487-499. doi: 10.1016/j. neuropharm.2016.02.006.

16. Collins M, An J, Peller D, Bowser R. Total protein is an effective loading control for cerebrospinal fluid western blots. J Neurosci Methods. 2015; 251:72-82. doi: 10.1016/j. jneumeth.2015.05.011. 